\title{
Feedback-controlled thermal therapy of tissues based on fiber Bragg grating thermometers
}

\author{
Sanzhar Korganbayev \\ Department of Mechanical \\ Engineering, \\ Politecnico di Milano \\ Milan, Italy \\ sanzhar.korganbayev@polimi.it
}

Alexey Wolf

Laboratory of Fiber Optics,

Institute of Automation and

Electrometry SB RAS,

Novosibirsk, Russia;

wolf@,iae.nsk.su

\author{
Annalisa Orrico \\ Department of Mechanical \\ Engineering, \\ Politecnico di Milano \\ Milan, Italy \\ annalisa.orrico@mail.polimi.it
}

Alexander Dostovalov Laboratory of Fiber Optics, Institute of Automation and

Electrometry SB RAS,

Novosibirsk, Russia;

dostovalov@iae.nsk.su

\author{
Leonardo Bianchi \\ Department of Mechanical \\ Engineering, \\ Politecnico di Milano \\ Milan, Italy \\ leonardo.bianchi@polimi.it
}

Paola Saccomandi

Department of Mechanical

Engineering,

Politecnico di Milano

Milan, Italy

paola.saccomandi@polimi.it

\author{
Martina De Landro \\ Department of Mechanical \\ Engineering, \\ Politecnico di Milano \\ Milan, Italy \\ martina.delandro@polimi.it
}

\begin{abstract}
Accurate treatment monitoring and control are essential factors to ensure safe and effective outcomes of thermal ablation therapies. In this work, we report a temperature feedback-controlled laser ablation system based on fiber Bragg grating (FBG) array measurements. A highly spatially resolved array of FBGs spaced at a distance of $0.01 \mathrm{~mm}$ was adopted to achieve accurate measurement of high-gradient temperature profiles during laser ablation in biological tissue. The temperature feedback-control based on FBG array measurements and on the subsequent laser current regulation was implemented to maintain stable peak temperature during ablation. The laser current was controlled based on the selection of threshold values, set for the maximum temperature in the laser-irradiated area, i.e., $43{ }^{\circ} \mathrm{C}, 48^{\circ} \mathrm{C}, 55{ }^{\circ} \mathrm{C}$, and $60^{\circ} \mathrm{C}$. The feedback-controlled system was validated comparing measured temperature maps during ablation and ablation results on the tissue surface. Finally, results suggest that our feedback system allows controlling the spatial extension of the ablated zone and preserving the maximum tissue temperature within useful ranges for a desired and optimal thermal ablation outcome.
\end{abstract}

Keywords-thermometry, temperature feedback-control, closed-loop, fiber optic thermometers, fiber Bragg grating sensors, thermal therapy, measurement instrumentation

\section{INTRODUCTION}

Thermal ablation techniques are minimally invasive approaches largely employed in interventional radiology for non-surgical candidates [1]. Cytotoxic temperatures (above 50 ${ }^{\circ} \mathrm{C}-60^{\circ} \mathrm{C}$ ) are needed to induce irreversible thermal damage within the target tumor. The main advantage of thermal ablation techniques over conventional treatments is the reduced invasiveness of the procedure, which leads to a shorter recovery time for the patient and, in general, a lower risk of post-surgical undesired effects. Moreover, the smallsize applicators used to increase the temperature in the treated region can reduce the risk of infection and discomfort for the patient. Among the available sources for thermal ablation techniques, laser has a unique advantage for clinical application, such as flexible fiber optic applicators that can deliver laser energy into deep-lying tumors [2], [3]. Additionally, the fiber optic applicator is compatible under

This project has received funding from the European Research Council (ERC) under the European Union's Horizon 2020 research and innovation programme (Grant agreement No. 759159). The work of A.W. and A.D. is supported by the State Budget of the Russian Federation (IAE project No. AAAAA17-117062110026-3). magnetic resonance guidance, which is among the most promising image-based thermometry techniques available nowadays [4]. The laser ablation (LA) has been clinically tested for tumor treatment in several organs, such as liver [5], pancreas [6], brain [7], [8], lung [9], prostate [10], breast tissue [11], and also for bones and localized treatments in the internal wall of hollow organs [12]-[15]

The laser-irradiated tissue target may have several regions presenting different biological effects. Typically, one region characterized by irreversible thermal damage (for $\mathrm{T}>50{ }^{\circ} \mathrm{C}$ ), surrounded by a region with reversible damage ( $\mathrm{T}$ comprised between $41^{\circ} \mathrm{C}$ and $45^{\circ} \mathrm{C}$ ) can be observed. Since temperature alone is not enough to define the thermal damage, but also time plays a meaningful role, some models have been proposed in the past to account for the influence of both parameters on the final therapy outcome. In particular, a previously defined model expresses the thermal dose as equivalent minutes of exposure at $43{ }^{\circ} \mathrm{C}$, which is commonly used to evaluate relative treatment effectiveness in tumor hyperthermia [16]. Nowadays, laser ablation is still suffering from some limitations, such as the non-uniform lesion obtained in the treated target and the lack of control of the treatment evolution and effective planning systems [17]. Indeed, an open-loop approach is usually implemented when laser settings are defined before the procedure based on tumor size and location, and these settings remain constant during the whole procedure

Some recent works have demonstrated that the use of temperature feedback is beneficial for the laser ablation outcome and for controlling the treated margins [18]. Most of these works regulate the laser power based on the tissue temperature measured with single-point sensors, such as thermocouples and thermistors. Even though these sensors are affordable and largely available in the labs, their main disadvantages are low spatial resolution and direct absorption of the laser light by metallic materials [19], [20].

Recently, our group has presented the first results on the use of fiber Bragg grating (FBG) sensors for regulating the laser power and controlling the treatment outcome [21], [22]. Fiber optic technology is interesting and largely used alternative to the traditional sensing approaches, especially for 
measurements in biomedical applications [23]-[25]. The biggest advantage owned by this sensing technology is the reduced invasiveness, achieved thanks to the multiplexing capability of the sensors. The compatibility of the FBG-based measurement systems with wavelength division multiplexing techniques makes these sensors appropriate for applications where large numbers of sensors are required. Indeed, a single fiber can host an array with a large number of sensors, easily more than 10 [26], [27]: one sensor can be used for the control, and the remaining sensors of the array can be employed for simultaneously measuring the temperature distribution within the tissue. Moreover, the material of the fiber optic sensors, usually quartz or plastic, makes them suitable to be used during laser-assisted procedures thanks to the absence of direct light absorption artifacts.

This work focuses on the improvement of laser ablation regulation by means of temperature measured with highly dense FBGs. The ON-OFF controlling algorithm was developed and implemented in LabVIEW, and tests with different set temperatures $\left(43^{\circ} \mathrm{C}, 48{ }^{\circ} \mathrm{C}, 55^{\circ} \mathrm{C}\right.$, and $\left.60{ }^{\circ} \mathrm{C}\right)$ were performed. The obtained temperature measurements and ablated tissue analysis validate the developed algorithm for treatment control.

\section{MATERIALS AND METHODS}

\section{A. Highly spatially-resolved Fiber Bragg Grating sensors}

FBGs employed in this work are intrinsic elements in single-mode fibers, where the index of refraction in the fiber core is periodically modulated by femtosecond laser processing [28]. The principle of signal measurements in FBG sensors is based on the response of the reflected wavelength due to tissue temperature changes induced by laser light. FBG arrays used for the experiments were fabricated using the femtosecond point-by-point writing technology [29] in singlemode optical fibers SM1500(9/125)P (Fibercore Ltd., Southampton, UK). Fig. 1a illustrated the reflected spectrum of the fabricated FBG array. All FBG reflection measurements were performed using Micron Optics si255 interrogation unit (Micron Optics, Atlanta, USA)

The single-mode fibers are coated with polyimide, which provides better temperature resistance to the sensing fiber, in comparison with acrylate [30]. The FBG arrays have the grating length of $1.19 \mathrm{~mm}$, and the edge-to-edge distance between gratings equal to $0.01 \mathrm{~mm}$. These properties have been chosen to have both high spatial resolution and the narrow spectral width of each Bragg wavelength peak. The total sensing length of each array is $50 \mathrm{~mm}$. The analysis of the difference between reflected Bragg wavelength with applied temperature change, $\lambda_{B, \Delta T}$, and the Bragg wavelength at a room temperature, $\lambda_{B}$, provides information about the Bragg wavelength shift, $\Delta \lambda_{B}$, and related temperature along the grating, $\Delta T$ :

$$
\frac{\Delta \lambda_{B}}{\lambda_{B}}=\frac{\lambda_{B, \Delta T}-\lambda_{B}}{\lambda_{B}}=\alpha \Delta T
$$

The calibration of the sensors was performed with Giussani Quartz thermostatic calibrator in the temperature range $30{ }^{\circ} \mathrm{C}-140{ }^{\circ} \mathrm{C}$, with a step of $10{ }^{\circ} \mathrm{C}$. For reference measurements, a PT100 thermistor was used. The results sensitivity is equal to $(7.43 \pm 0.01) \cdot 10^{-6}{ }^{\circ} \mathrm{C}^{-1}$.
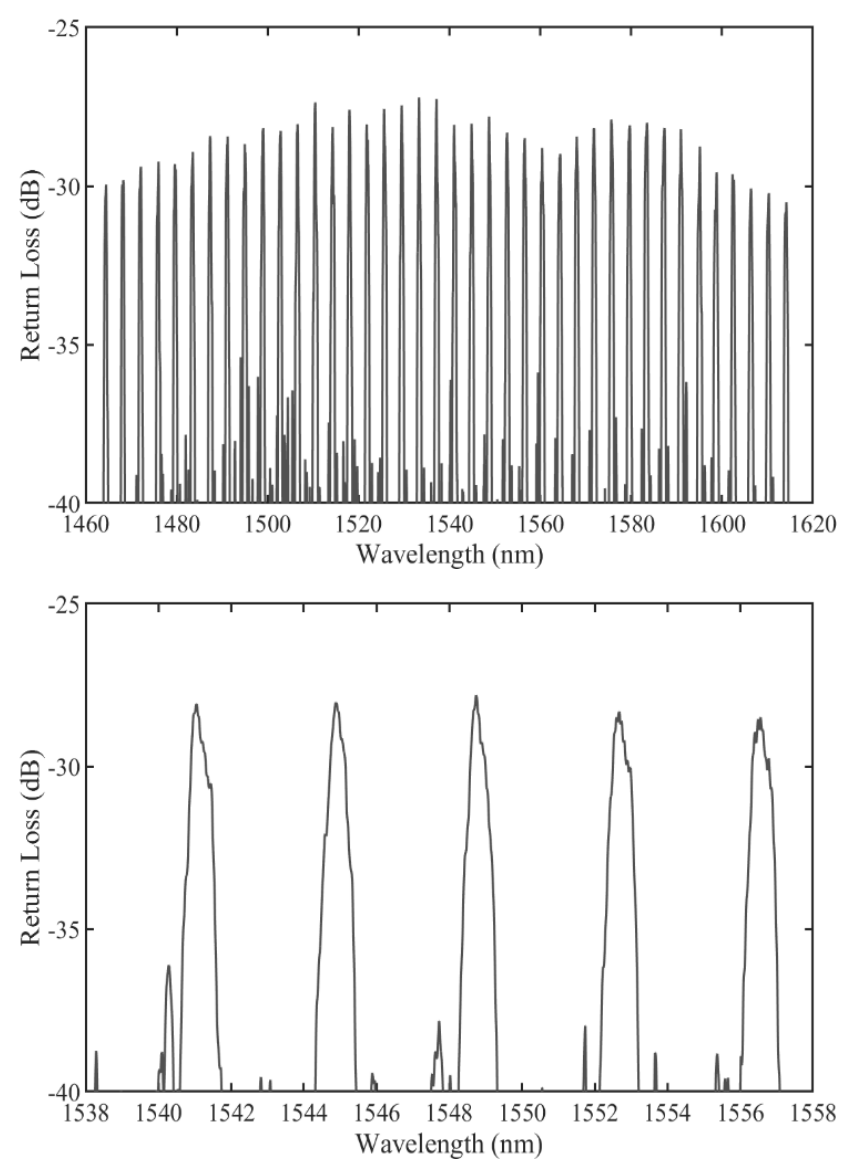

Fig. 1. (top) Reflection spectrum of the 40-FBG array fabricated with the femtosecond point-by-point writing technology. (bottom) Zoom of the spectrum on the central gratings.

\section{B. Thermal ablation experimental setup}

For ablation experiments, the fresh porcine liver obtained from a local butcher was used as a target tissue, the liver was at room temperature exactly before the start of the experiments.

Thermal ablation was performed with a near-infrared diode laser. Laser energy was delivered on the ex-vivo porcine liver surface in a superficial manner (laser beam is perpendicular to the abated surface) using collimator (OZ Optics Ltd., Ottawa, Canada) (Fig. 2). The diameter of the laser spot on the liver surface was $1 \mathrm{~cm}$, and the laser power was $2 \mathrm{~W}$, and the related power density was $2.55 \mathrm{~W} / \mathrm{cm}^{2}$. The spot of the laser was focused on the central sensing region of the FBG array, which was adjusted on the surface of the organ.

\section{Temperature feedback-controlled strategy}

The developed control algorithm utilizes an ON-OFF logic, which aims at controlling the current of the laser according to the measured temperature. As depicted in Fig. 3, ablation starts at room temperature $\mathrm{T}_{0}\left(\sim 23{ }^{\circ} \mathrm{C}\right)$, the laser is turned OFF when the maximum measured temperature exceeds the set temperature $\mathrm{T}_{\mathrm{S}}$. Then the maximum temperature is controlled with ON-OFF logic until the clinician stops the treatment. In order to prevent overheating of the laser, comparison of set temperature and the measured maximum temperature is executed each $\Delta \tau$ seconds.

Preliminary evaluations of the optimal value for the comparison period shows that $\Delta \tau=1 \mathrm{~s}$ provides smooth temperature control, that leads to a spatially confined ablation 
area [21]; hence, $\Delta \tau$ was set to $1 \mathrm{~s}$ for all experiments. For the experimental part, controlled ablation with different $\mathrm{T}_{\mathrm{S}}$ values was performed: $43{ }^{\circ} \mathrm{C}, 48{ }^{\circ} \mathrm{C}, 55{ }^{\circ} \mathrm{C}$, and $60{ }^{\circ} \mathrm{C}$. Each experiment was repeated three times. The LabVIEW program was designed to receive FBG reflected spectra from the Micron Optics interrogation unit, evaluate and plot the temperature profile along the FBG array, and adjust the laser current based on the maximum temperature measured in realtime. Experiments were also performed in the absence of power regulation in order to compare the effects and benefits of the temperature feedback-control with the standard procedure.

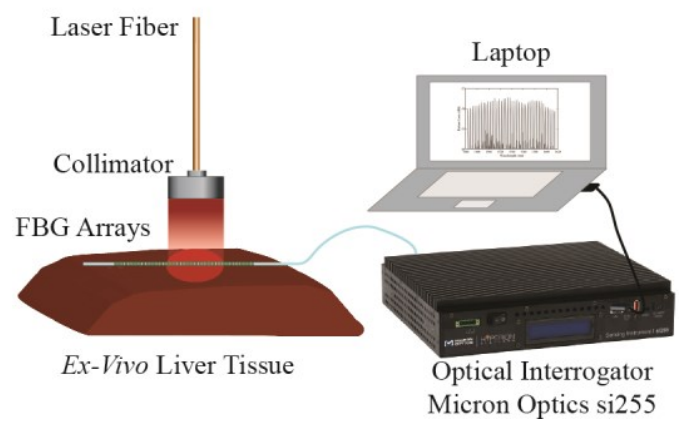

Fig. 2. Experimental setup: LA of ex-vivo liver is perfromed in superficial manner. LA control (LabVIEW proogram on laptop) is based on maximum temperature measured by FBG array during ablation.

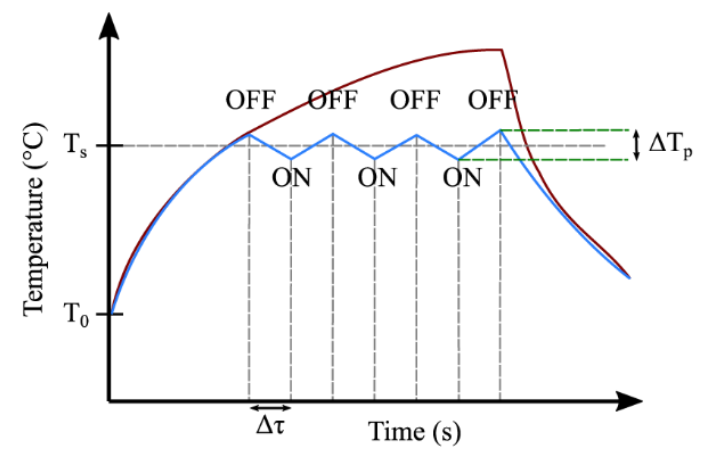

Fig. 3. Schematics of peak temperature evolution during LA for: noncontrolled ablation (red line) and ON-OFF controlled ablation (blue line). $\mathrm{T}_{\mathrm{S}}$ is the set temperature, $\mathrm{T}_{0}$ is the initial temperature, $\Delta \tau$ is the time between the moments when the algorithm compares current and set temperatures, $\Delta \mathrm{T}_{\mathrm{p}}$ is the temperature oscillation interval.

\section{RESULTS}

As shown in Fig. 4, the thermal effects of the regulation strategy are firstly evaluated in terms of temperature evolution measured by the central FBG (maximum FBG in Fig. 4a) and temperature profiles at specified distances from the maximum (i.e., $-4.8 \mathrm{~mm},-2.4 \mathrm{~mm}, 2.4 \mathrm{~mm}$ and $4.8 \mathrm{~mm}$, Figs. $4 \mathrm{~b}-\mathrm{f}$ ).

Uncontrolled trends and temperature evolutions obtained with different $T_{S}$ are clearly distinguishable. The uncontrolled ablation tests experience maximum temperatures of $76.7^{\circ} \mathrm{C}$, whereas in the controlled cases, the threshold is followed. For higher values of $T_{S}$, temperature spikes are more observable, and the temperature oscillation intervals are higher. By defining the temperature oscillations $\Delta T_{p}$ as the mean difference between the peak and the valley of the oscillating trends across $T_{S}$, the following results are obtained: $5.8 \pm 0.8$

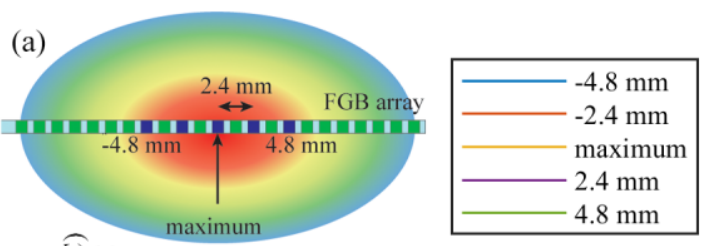

(b)

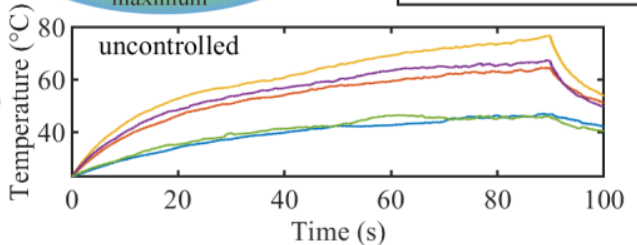

(c)

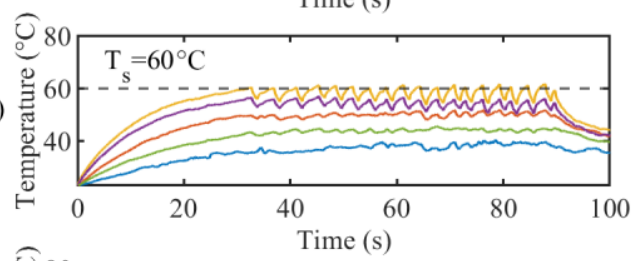

(d)

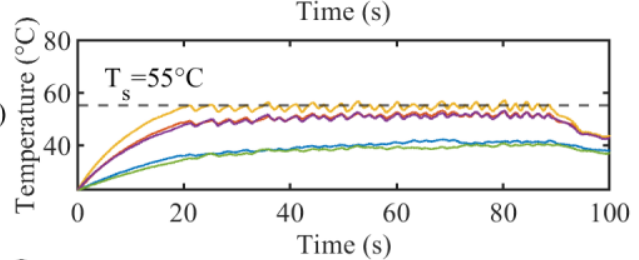

(e)

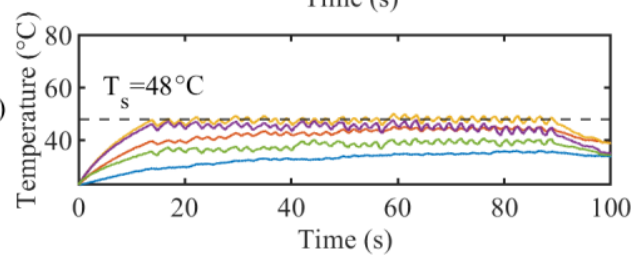

(f)

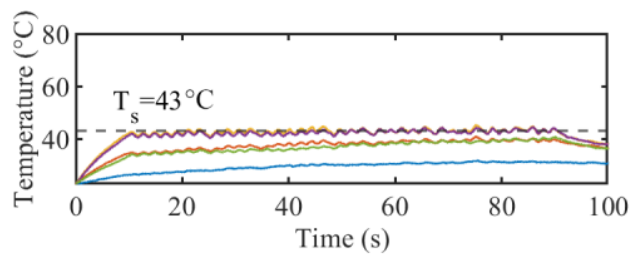

Fig. 4. Maximum temperature profile and profiles at specific distances from it: (a) schematics of the gratings used for graphs (blue gratings); (b) uncontrolled ablation and controlled ablation for the set temperature $T_{S}$ equal to (c) $60{ }^{\circ} \mathrm{C}$; (d) $55^{\circ} \mathrm{C}$; (e) $48^{\circ} \mathrm{C}$, and (f) $43{ }^{\circ} \mathrm{C}$.

${ }^{\circ} \mathrm{C}, 2.8 \pm 0.7{ }^{\circ} \mathrm{C}, 2.5 \pm 0.4{ }^{\circ} \mathrm{C}$, and $2.0 \pm 0.6{ }^{\circ} \mathrm{C}$ for $T_{S}=60{ }^{\circ} \mathrm{C}, 55$ ${ }^{\circ} \mathrm{C}, 48{ }^{\circ} \mathrm{C}$ and $43{ }^{\circ} \mathrm{C}$, respectively. We can observe that the temperature oscillation interval decreases with distance from the maximum temperature location for controlled ablation cases because at a major distance from the laser spot the temperature increases only due to heat conduction, which has a smoother response to an ON-OFF control.

Fig. 5 shows the change of the width of the hyperthermia zone $\left(>43{ }^{\circ} \mathrm{C}\right)$ in time, during uncontrolled ablation (Fig. 5a) and controlled ones with $\mathrm{T}_{\mathrm{S}}=60^{\circ} \mathrm{C}, 55^{\circ} \mathrm{C}, 48^{\circ} \mathrm{C}$ and, $43{ }^{\circ} \mathrm{C}$ for the first trial (Figs. 5b-e). The zone is small and not continuous for $T_{S}=43{ }^{\circ} \mathrm{C}$ (Fig. 5.e), because when $T_{S}$ was reached, the laser was set immediately OFF, not allowing broadening of the hyperthermia zone. 


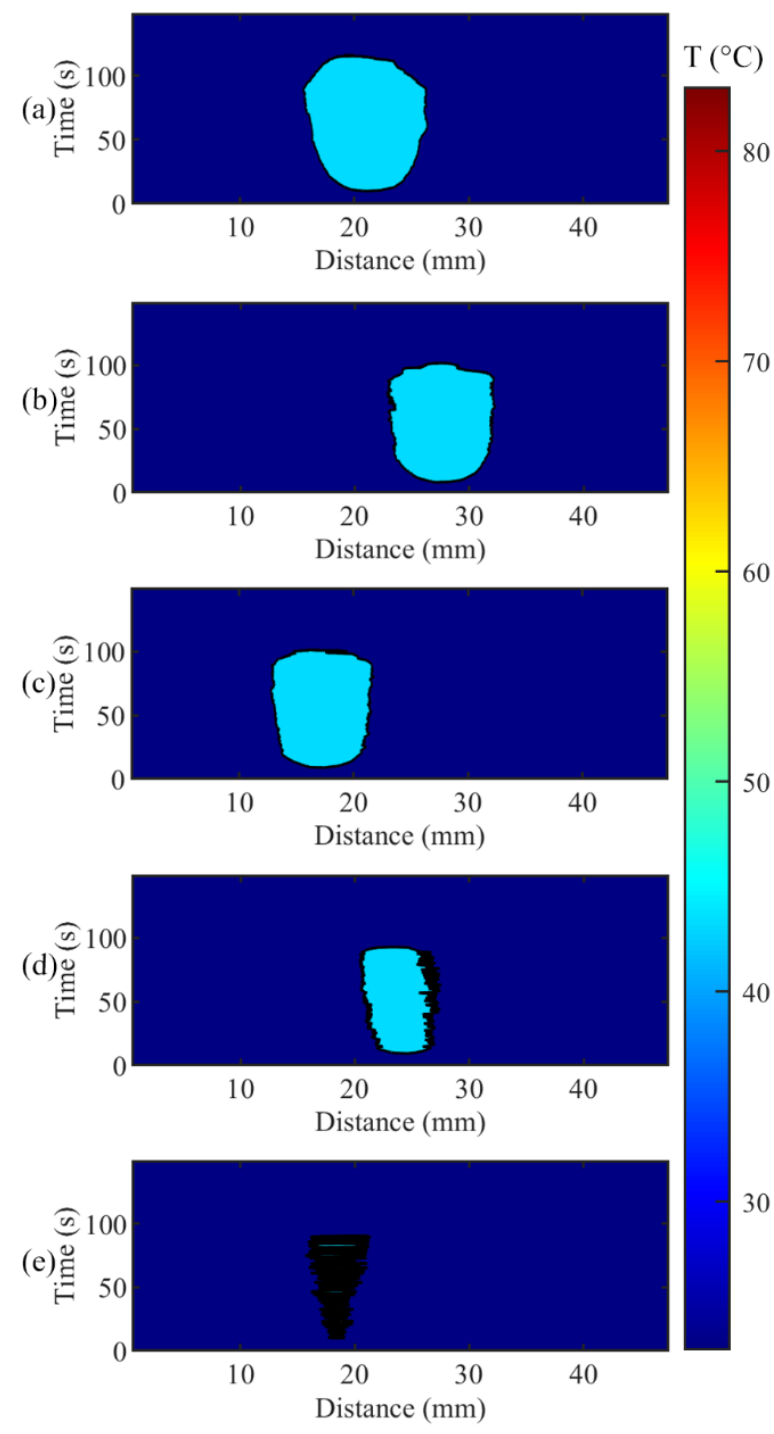

Fig. 5. Two-dimensional contour maps (distance along the sensor vs. time) with $43{ }^{\circ} \mathrm{C}$ threshold during (a) uncontrolled ablation; and controlled ablation for set temperature $T_{S}$ equal to (b) $60{ }^{\circ} \mathrm{C}$; (c) $55{ }^{\circ} \mathrm{C}$; (d) $48^{\circ} \mathrm{C}$, and (e) $43{ }^{\circ} \mathrm{C}$.

Results of the ablations with different $T_{S}$ values are presented in Fig. 6. As expected, the uncontrolled ablation has the largest ablated area as well as the higher damage (showing the importance of LA control to contain the damage to the selected area). While $T_{S}=60^{\circ} \mathrm{C}$ and $55^{\circ} \mathrm{C}$ cases show ablation regions with regular borders and restrained damage. Specifically, the visible damage in the RGB images is localized in the area of interest compared to the uncontrolled case, with lower damage for smaller temperature. For $T_{S}=48$ ${ }^{\circ} \mathrm{C}$ and $43{ }^{\circ} \mathrm{C}$, the ablation areas look undefined with no significant tissue changes visible in the images. (a)

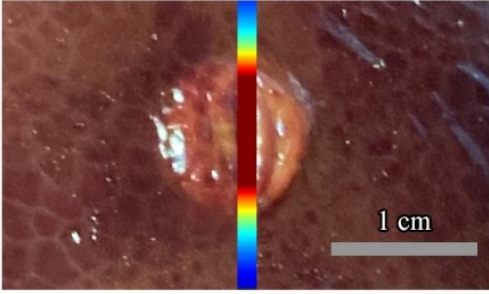

(b)

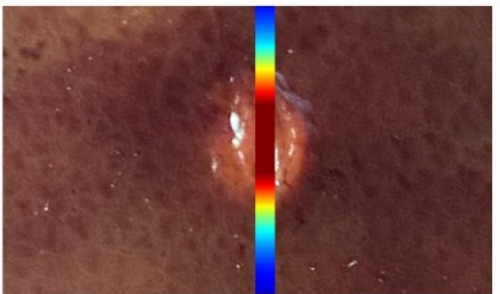

(c)

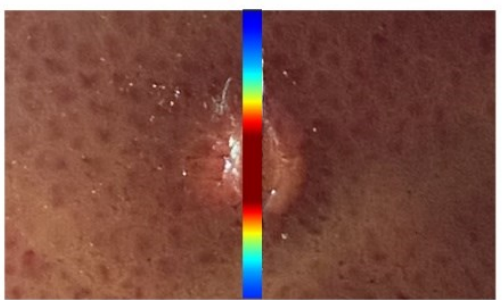

(d)

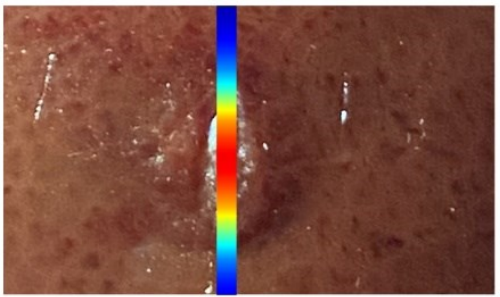

(e)

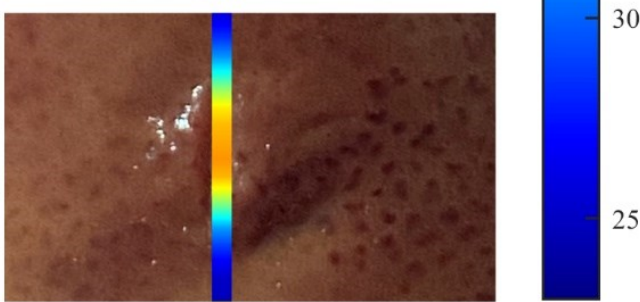

Fig. 6. Treated surface tissue after ablation experiments and maximum temperature profiles along the sensor (at the end of ablation) for: (a) uncontrolled ablation; and controlled ablation for set temperature $T_{S}$ equal to (b) $60{ }^{\circ} \mathrm{C}$; (c) $55^{\circ} \mathrm{C}$, (d) $48^{\circ} \mathrm{C}$ and (e) $43{ }^{\circ} \mathrm{C}$. Thermal maps are shown in the correspondence of the fiber sensors' positions.

\section{DISCUSSION AND CONCLUSIONS}

This work presents a multi-threshold temperature feedback-controlled laser ablation based on quasi-distributed FBG sensors. The main objective of the work is preventing undesired effects on the healthy tissues and guaranteeing proper ablation of the interested region. For temperaturebased control of diode laser, the ON-OFF control logic was implemented to control the maximum temperature during ablation. The tissue temperature was measured with arrays of 40 FBGs, fabricated with high spatial resolution properties and high-temperature resistance polyimide coating. Experiments with different set temperatures were performed: $43^{\circ} \mathrm{C}, 48^{\circ} \mathrm{C}, 55^{\circ} \mathrm{C}$, and $60{ }^{\circ} \mathrm{C}$, and compared to the treatment without power regulation. The developed algorithm was evaluated in terms of measured thermal maps during an ex vivo porcine liver ablation and as well as of surface tissue 
changes after ablation. In addition, analysis of the evaluation of the treated margins was performed by assessment of hyperthermia zone's width, which is defined by regions of the tissue exposed to temperatures higher than $43^{\circ} \mathrm{C}$. This value is important for LA analysis, because it can be used in the Cumulative Equivalent Minutes at $43{ }^{\circ} \mathrm{C}$ model (CEM43) to evaluate thermal dose of the treatment and it's effect on the tissue [16].

The developed experimental setup and the obtained results prove the efficacy of FBG array-based LA control techniques. The main advantage of FBG arrays over other traditional temperature monitoring methods for LA control is the quasidistributed sensing capability, which makes them well suited for thermal ablation monitoring, as it allows for high-spatialresolution temperature distribution measurements along the fiber [26], [31], [32]. In addition, inherent advantages of fiber sensing, such as electromagnetic immunity, miniature size, low laser light absorption, and biocompatibility, make FBGs well-suited for LA applications [33]. The results of the work show that set values of temperature-based feedback control affect the size of the ablated region and overall tissue properties after treatment. The set temperatures were contained within ranges corresponding to specific thermal states of biological tissues. These evidences encourage new analysis towards the optimal control strategy. Indeed, solutions for reducing the temperature oscillations could be put in place.

\section{REFERENCES}

[1] S. N. Goldberg, G. S. Gazelle, and P. R. Mueller, "Thermal ablation therapy for focal malignancy: A unified approach to underlying principles, techniques, and diagnostic imaging guidance," American Journal of Roentgenology. 2000, doi: 10.2214/ajr.174.2.1740323

[2] F. M. Di Matteo et al., "Feasibility of EUS-guided Nd:YAG laser ablation of unresectable pancreatic adenocarcinoma," Gastrointest. Endosc., vol. 88, no. 1, pp. 168-174.e1, Jul. 2018, doi: 10.1016/j.gie.2018.02.007.

[3] R. J. Stafford, D. Fuentes, A. A. Elliott, J. S. Weinberg, and K. Ahrar, "Laser-Induced Thermal Therapy for Tumor Ablation," Crit. Rev. Biomed. Eng., vol. 38, no. 1, pp. 79-100, 2010, doi: 10.1615/CritRevBiomedEng.v38.i1.70.

[4] M. De Landro et al., "Fiber bragg grating sensors for performance evaluation of fast magnetic resonance thermometry on synthetic phantom," Sensors (Switzerland), 2020, doi: 10.3390/s20226468.

T. J. Vogl, R. Straub, K. Eichler, O. Söllner, and M. G. Mack, "Colorectal Carcinoma Metastases in Liver: Laser-induced Interstitial Thermotherapy-Local Tumor Control Rate and Survival Data," Radiology, vol. 230, no. 2, pp. 450-458, Feb. 2004, doi: 10.1148/radiol.2302020646.

[6] P. Saccomandi et al., "Theoretical Analysis and Experimental Evaluation of Laser-Induced Interstitial Thermotherapy in Ex Vivo Porcine Pancreas," IEEE Trans. Biomed. Eng., vol. 59, no. 10, pp. 2958-2964, Oct. 2012, doi: 10.1109/TBME.2012.2210895.

[7] H.-J. Schwarzmaier et al., "MR-guided laser-induced interstitial thermotherapy of recurrent glioblastoma multiforme: preliminary results in 16 patients," Eur. J. Radiol., vol. 59, no. 2, pp. 208-215, 2006.

[8] L. K. Swartz, K. G. Holste, M. M. Kim, A. Morikawa, and J. Heth, "Outcomes in Patients Treated with Laser Interstitial Thermal Therapy for Primary Brain Cancer and Brain Metastases," Oncologist, vol. 24, no. 12, pp. e1467-e1470, Dec. 2019, doi: 10.1634/theoncologist.2019-0213.

[9] J. A. S. Brookes, W. R. Lees, and S. G. Bown, "Interstitial laser photocoagulation for the treatment of lung cancer.," Am. J.
Roentgenol., vol. 168, no. 2, pp. 357-358, Feb. 1997, doi: 10.2214/ajr.168.2.9016205.

[10] R. Muschter, "Laser-induced interstitial thermotherapy of benign prostatic hyperplasia and prostate cancer," in Medical Applications of Lasers II, Dec. 1994, vol. 2327, pp. 287-292, doi: $10.1117 / 12.197575$.

[11] Y. Kerbage et al., "Laser interstitial thermotherapy application for breast surgery: Current situation and new trends," The Breast, vol. 33, pp. 145-152, Jun. 2017, doi: 10.1016/j.breast.2017.03.016.

[12] A. L. Gough-Palmer and W. M. Witold Gedroyc, "Laser ablation of hepatocellular carcinoma - A review," World Journal of Gastroenterology. 2008, doi: 10.3748/wjg.14.7170.

[13] S. Ambastha, S. Pant, S. Umesh, V. Vazhayil, and S. Asokan, "Feasibility Study on Thermography of Embedded Tumor Using Fiber Bragg Grating Thermal Sensor," IEEE Sens. J., 2020, doi: 10.1109/JSEN.2019.2950973.

[14] P. Saccomandi et al., "Laser ablation in biliary tree: analysis of the intraductal and superficial thermal effects during the treatment," 2019, doi: 10.1109/embc.2019.8856313.

[15] R. Rea et al., "Endoluminal Nd:YAG laser application in ex vivo biliary porcine tissue," Lasers Med. Sci., 2017, doi: 10.1007/s10103-017-2264-0.

[16] P. S. Yarmolenko et al., "Thresholds for thermal damage to normal tissues: an update.," Int. J. Hyperthermia, vol. 27, no. 4, pp. 32043, Jun. 2011, doi: 10.3109/02656736.2010.534527.

[17] P. Saccomandi, E. Schena, and C. M. Pacella, "New Horizons for Laser Ablation: Nanomedicine, Thermometry, and Hyperthermal Treatment Planning Tools," in Image-guided Laser Ablation, Springer, 2020, pp. 145-151.

[18] V. N. Tran, V. G. Truong, S. Jeong, and H. W. Kang, "Computational analysis of linear energy modulation for laser thermal coagulation," Biomed. Opt. Express, 2018, doi: 10.1364/boe.9.002575.

[19] P. H. Mollert, L. Lindbergt, P. H. Henriksson, and K. Tranberg, "Temperature control and light penetration in a feedback interstitial laser thermotherapy system," vol. 12, no. 1, pp. 49-63, 1996.

[20] K. Ivarsson, J. Olsrud, and C. Sturesson, "Feedback Interstitial Diode Laser ( $805 \mathrm{~nm}$ ) Thermotherapy System: Ex Vivo Evaluation and Mathematical Modeling With One and FourFibers," vol. 96, no. September 1997, pp. 86-96, 1998.

[21] S. Korganbayev, R. Pini, A. Orrico, A. Wolf, A. Dostovalov, and P. Saccomandi, "Towards temperature-controlled laser ablation based on fiber Bragg grating array temperature measurements," in 2020 IEEE International Workshop on Metrology for Industry 4.0 \& IoT, 2020, pp. 268-272.

[22] S. Korganbayev et al., "Closed-Loop Temperature Control Based on Fiber Bragg Grating Sensors for Laser Ablation of Hepatic Tissue," Sensors, vol. 20, no. 22, p. 6496, 2020.

[23] D. Lo Presti et al., "Fiber bragg gratings for medical applications and future challenges: A review," IEEE Access, 2020, doi: 10.1109/ACCESS.2020.3019138.

[24] K. A. Tomyshev, D. K. Tazhetdinova, E. S. Manuilovich, and O. V. Butov, "High-resolution fiber optic surface plasmon resonance sensor for biomedical applications," J. Appl. Phys., 2018, doi: $10.1063 / 1.5045180$.

[25] L. Bianchi et al., "Fiber Bragg Grating Sensors for Thermometry during Gold Nanorods-mediated Photothermal Therapy in Tumor Model," in 2020 IEEE Sensors, Oct. 2020, pp. 1-4, doi: 10.1109/SENSORS47125.2020.9278580.

[26] F. Morra et al., "Spatially resolved thermometry during laser ablation in tissues: Distributed and quasi-distributed fiber opticbased sensing," Opt. Fiber Technol., vol. 58, p. 102295, 2020.

[27] S. Korganbayev, M. De Landro, F. Morra, A. Cigada, and P. Saccomandi, "Fiber Optic Sensors for Distributed and Quasidistributed Temperature Measurement," in 2020 IEEE Sensors, 
2020, pp. 1-4.

[28] C. R. Liao and D. N. Wang, "Review of femtosecond laser fabricated fiber Bragg gratings for high temperature sensing," Photonic Sensors, 2013, doi: 10.1007/s13320-012-0060-9.

[29] A. V Dostovalov, A. A. Wolf, A. V Parygin, V. E. Zyubin, and S. A. Babin, "Femtosecond point-by-point inscription of Bragg gratings by drawing a coated fiber through ferrule," Opt. Express, vol. 24, no. 15, pp. 16232-16237, 2016.

[30] L. Huang, R. S. Dyer, R. J. Lago, A. A. Stolov, and J. Li, "Mechanical properties of polyimide coated optical fibers at elevated temperatures," in Optical Fibers and Sensors for Medical Diagnostics and Treatment Applications XVI, Mar. 2016, p. 97020Y, doi: 10.1117/12.2210957.
[31] S. Korganbayev et al., "Highly dense FBG arrays for millimeterscale thermal monitoring during nanocomposite-enhanced laser ablation," in Optical Sensing and Detection VI, 2020, vol. 11354, p. $113540 \mathrm{G}$.

[32] L. Bianchi, S. Korganbayev, A. Orrico, M. De Landro, and P. Saccomandi, "Quasi-distributed fiber optic sensor-based control system for interstitial laser ablation of tissue: theoretical and experimental investigations," Biomed. Opt. Express, vol. 12, no. 5, p. 2841, May 2021, doi: 10.1364/BOE.419541.

[33] A. Othonos, K. Kalli, and G. E. Kohnke, "Fiber Bragg gratings: Fundamentals and applications in telecommunications and sensing," Phys. Today, vol. 53, no. 5, p. 61, 2000. 